University of Nebraska - Lincoln

DigitalCommons@University of Nebraska - Lincoln

5-18-1998

\title{
Pressure lonization and Line Merging in Strongly Coupled Plasmas Produced by $100-$ fs Laser Pulses
}

\author{
M. Nantel \\ University of Michigan, Ann Arbor \\ G. Ma \\ University of Michigan, Ann Arbor \\ S. Gu \\ University of Michigan, Ann Arbor \\ C.Y. Côté \\ University of Michigan, Ann Arbor \\ J. Itatani \\ University of Michigan, Ann Arbor \\ See next page for additional authors
}

Follow this and additional works at: https://digitalcommons.unl.edu/physicsumstadter

Part of the Physics Commons

Nantel, M.; Ma, G.; Gu, S.; Côté, C.Y.; Itatani, J.; and Umstadter, Donald, "Pressure Ionization and Line Merging in Strongly Coupled Plasmas Produced by 100-fs Laser Pulses" (1998). Donald Umstadter Publications. 25.

https://digitalcommons.unl.edu/physicsumstadter/25

This Article is brought to you for free and open access by the Research Papers in Physics and Astronomy at DigitalCommons@University of Nebraska - Lincoln. It has been accepted for inclusion in Donald Umstadter Publications by an authorized administrator of DigitalCommons@University of Nebraska - Lincoln. 


\section{Authors}

M. Nantel, G. Ma, S. Gu, C.Y. Côté, J. Itatani, and Donald Umstadter

This article is available at DigitalCommons@University of Nebraska - Lincoln: https://digitalcommons.unl.edu/ physicsumstadter/25 


\title{
Pressure Ionization and Line Merging in Strongly Coupled Plasmas Produced by 100-fs Laser Pulses
}

\author{
M. Nantel, G. Ma, S. Gu, C. Y. Côté, J. Itatani, and D. Umstadter \\ Center for Ultrafast Optical Science, University of Michigan, 1006 IST Building, 2200 Bonisteel, Ann Arbor, Michigan 48109-2099
}

(Received 8 October 1997)

\begin{abstract}
Time- and space-resolved extreme ultraviolet spectra of carbon plasmas, created with 100-fs laser pulses, are obtained with the novel technique of picosecond jitter-free streak-camera averaging. Spectroscopic diagnostics indicate electron densities and temperatures evolving from $10^{23}$ to $10^{21} \mathrm{~cm}^{-3}$ and 80 to $50 \mathrm{eV}$, respectively, implying less than one particle in a Debye sphere at early times. The emission reveals conditions of extreme pressure ionization and line merging. Comparisons of the experimental spectra with numerical simulations validate the use of the Inglis-Teller limit for line merging, and confirm that pressure ionization models based on the Debye-Huckel potential are inapplicable in such strongly coupled plasmas. [S0031-9007(98)06112-2]
\end{abstract}

PACS numbers: 52.70.Kz, 52.25.Jm, 52.40.Nk, 52.50.Jm

Strongly coupled plasmas (SCPs) are found in stellar and Jovian planet interiors [1], inertial confinement fusion (ICF) pellets [2], and are essential for a thorough understanding of compressed material equations of state [3]. These plasmas are characterized by high densities and/or relatively low temperatures, such that the Coulomb potential energy between the particles exceeds their kinetic energy, and thus the coupling parameter $\Gamma=Z^{2} e^{2} / r_{i} k T$ (where $r_{i}$ is the ion-sphere radius) is larger than one. Unfortunately, relatively little experimental work on strongly coupled plasmas [3-5] has been done up to now, as these studies are rendered difficult by the need to simultaneously obtain and diagnose high-density/low-temperature conditions. This is particularly true for subpicosecond laserproduced plasmas, which are increasingly important in applications such as fast-ignitor ICF, ultrafast $\mathrm{x}$-ray sources, and ion acceleration. Pressure ionization and line merging are two spectroscopic effects arising from the deformation of the ionic potential by the plasma fields in SCPs [6]; although both have been used for density diagnostics [5,7], independent plasma diagnostics must be used to ensure their validity in the strongly coupled regime. In this paper, we present extreme ultraviolet (XUV) spectra emitted from a strongly coupled laserproduced plasma, making use of two novel experimental techniques: (1) A high-contrast, high-seed injection CPA short-pulse laser [8] to achieve nearly solid density, and (2) a jitter-free $\mathrm{x}$-ray streak camera $[9,10]$ to achieve both a high signal-to-noise ratio and ultrahigh temporal resolution (ps). This combination: short laser pulses and high temporally and spatially resolved spectroscopy, makes it possible to study the dynamics of SCPs over a wide range of densities and temperatures, which makes for an interesting testbed for atomic physics models of such plasmas. We follow the change in the observed series limits that occurs as carbon is heated and then decompresses over a range of electron density $n_{e}$ and temperature $T_{e}$ extending from $10^{23}$ to $10^{21} \mathrm{~cm}^{-3}$ and 80 to $50 \mathrm{eV}$, respectively, which corresponds to $1.5>\Gamma>0.5$. Making use of independent plasma diagnostics over this large range of parameter space, we demonstrate the breakdown of the Debye-Huckel model for pressure ionization, and establish the validity of the Inglis-Teller limit for line-merging predictions.

The experiments were conducted with a $10-\mathrm{Hz}$, 100-fs Ti:sapphire laser. Pulses of $50 \mathrm{~mJ}$ in energy at $780 \mathrm{~nm}$ were focused with a $\mathrm{MgF}_{2}$ lens to an intensity of $10^{17} \mathrm{~W} / \mathrm{cm}^{2}$ on rotating solid carbon disk targets. The use of subpicosecond pulses has the advantage of depositing the energy impulsively, in a thin target layer. Thus, the measurements are conducted after the laser pulse, in a freely decompressing ionized material. The emission spectra from the target were recorded with a grazing-incidence flat-field imaging XUV spectrometer coupled to an $\mathrm{X}$-ray streak camera with a charge-coupled device (CCD) readout, for a spatial resolution of $80 \mu \mathrm{m}$ in the direction normal to the target plane and a spectral resolution of $0.15 \AA$. The subpicosecond x-ray streak camera [10] was coupled to a jitter-free averaging sweep system [9], consisting of two photoconductive switches triggered by part of the laser beam to sweep the plates of the camera at $10 \mathrm{~Hz}$, in synchronization with the target emission. This novel technique-used for the first time here for spectroscopy - significantly extends the dynamic range of the streak camera and increases the signal-tonoise ratio. We were able to average the XUV spectrum over 600 shots with a temporal resolution of $4 \mathrm{ps}$, limited by a residual jitter of \pm 2 ps. The laser contrast was improved by a factor of more than 100 over the typical figure of $10^{5}$ for Ti:sapphire lasers through high-energy seeding of clean pulses in the regenerative amplifier [8]. This new technique delivers a laser contrast of $>5 \times 10^{7}$ to allow the deposition of the laser energy at solid target densities without the energy losses incurred by frequency doubling.

We present data from the $80-\mu \mathrm{m}$ region closest to the target plane. Figure 1 shows three spectra between 25 and $37 \AA$ taken at 0,50 , and 225 ps. These lineouts are 


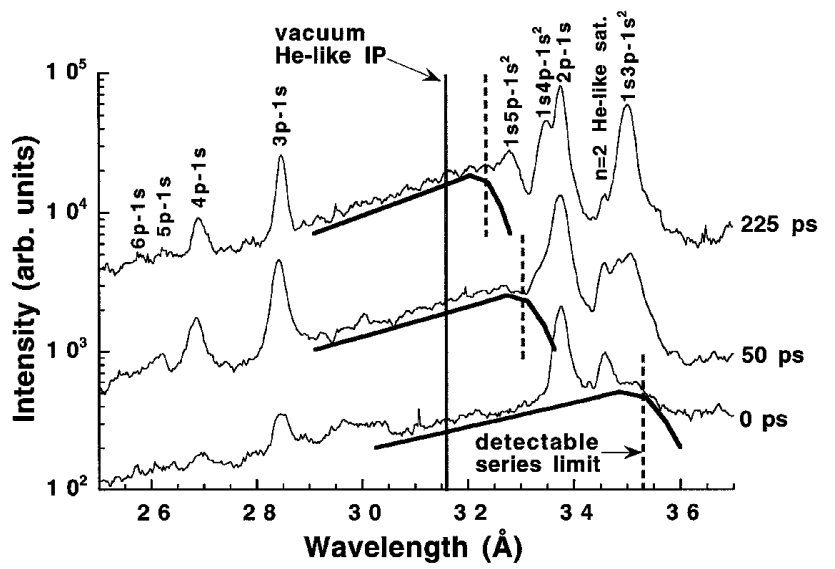

FIG. 1. Lineouts from the time-resolved spectra taken at $t=0$, 50, and $225 \mathrm{ps}$. The He-like $n p 1 s-1 s^{2}(n>3)$ and $\mathrm{H}$-like $n p-1 s$ line emissions are identified, as well as the $n=2$ He-like satellites to the H-like $2 p-1 s$ line. The free-bound continuum and the detectable series limit are drawn on each lineout. Considerable He-like $2 p 1 s-1 s^{2}$ emission at $40.268 \AA$ was seen in each spectrum, but is not included here for better emphasis on the spectral region between 25 and $37 \AA$. The three lineouts are offset vertically for better viewing.

integrated over $4 \mathrm{ps}$, and $t=0 \mathrm{ps}$ is arbitrarily defined as the time of the start of the emission. The principal emission lines of the H-like and He-like series are identified, as well as the $n=2 \mathrm{He}$-like satellites to the H-like $2 p-1 s$ line. There is considerable free-bound continuum radiation ending at the He-like series limit near the $\mathrm{H}$-like $2 p-1 s$ line. The vacuum ionization potential (IP) of the He-like ion is marked at $31.62 \AA(392.09 \mathrm{eV})$ [11], and the He-like radiative recombination continuum and edge are sketched in bold lines. The position of the detectable series limit (marked by vertical dashed lines for each spectrum) is determined to $\approx 0.4-0.7 \AA$, limited by the presence of the unmerged bound-bound emission lines masking its exact position. This detectable He-like series limit is measured to move from $35.2 \AA(352.5 \mathrm{eV})$ at $0 \mathrm{ps}$, to $33.1 \AA$ $(374.3 \mathrm{eV})$ at $50 \mathrm{ps}$, and to $32.6 \AA(380.9 \mathrm{eV})$ at $225 \mathrm{ps}$. The separation between the vacuum IP and the observed edge for each spectrum indicates the combined effect on the spectrum of pressure ionization and line merging, which can move the observable series limit by as much as $40 \mathrm{eV}$, or past the He-like $3 p-1 s$ resonance line. No unmerged resonance lines from the He-like series are detected at 0 ps save the strong $2 p-1 s$ transitions (not shown in Fig. 1 for better viewing of the wavelength range near the series limit), and all the readily identifiable satellites to the H-like $2 p-1 s$ line originate from $n=2$ doubly excited states. As the plasma evolves with time, the density - and hence the plasma field effects - gradually decrease and the ionic potential is less and less perturbed: Identifiable line emissions from higher- $n$ states reappear gradually, as is observed at $50 \mathrm{ps}$ (He-like $1 s 3 p-1 s^{2}$ clearly present and $1 s 4 p-1 s^{2}$ visible in the H-like $2 p-1 s$ short-wavelength shoulder) and at 225 ps (He-like $1 s 4 p-1 s^{2}$ and $1 s 5 p-1 s^{2}$ now clearly identifiable). A similar trend is observed in the higher- $n \mathrm{H}$-like emission lines, with the strengthening of the $3 p-1 s$ and $4 p-1 s$ lines, and the appearance of the $5 p-1 s$ and $6 p-1 s$ in the later lineouts, despite the falling temperature evident from the steepening of the continuum slope.

There is still considerable debate as to what formalism should be used to best describe pressure ionization and line merging [12-15], but the more widely used pressure ionization models in numerical simulations and analysis of experimental spectra are the ion-cell, Debye-Huckel, and Stewart-Pyatt models [5,7,16-19]. These define a sharp cutoff for the highest existing principal quantum number $n$ from an average static electric field without considering the fluctuating microfields that will broaden the levels near the continuum, and must be used in conjunction with a good Stark broadening model to correctly reproduce the experimental spectra. Alternatively, the Inglis-Teller limit [20] predicts the density at which two adjacent levels merge into an apparent continuum, and approximatively takes care of microfield fluctuations and line merging. All these models predict a lowering of the ionization potential with increasing density, and a movement of the free-bound emission edges and the detectable series limits to longer wavelengths, as seen in Fig. 1. Direct experimental evidence of the amount of pressure ionization is usually hidden by the line merging which occurs at lower principal quantum numbers $n$. It is therefore difficult to determine which pressure ionization model is best [19]. Experiments providing precise data for model validation are rare - and the few that have been reported involved plasmas that, in addition to their limited density and temperature ranges, were restricted to the weakly coupled regime $(\Gamma \leq 0.2)[15,21]$.

In our analysis, electron temperature and density diagnostics were obtained from the experimental spectra. We first established the electron temperature by the slope of the He-like continuum. We then generated artificial spectra using steady-state non-local-thermodynamic equilibrium (non-LTE) FLY simulations [18] and found matches for the experimental spectra by varying the density in the simulation. This was done for eight lineouts at times $0,14,25$, $50,75,125,175$, and 225 ps after the start of the emission. The artificial spectra includes instrumental broadening, pressure ionization, Stark broadening, and opacity. The different models of pressure ionization that were used for the generation of the excited state populations and artificial spectra were the Stewart-Pyatt model (already included in FLY), and the ion-cell and Debye-Huckel models (implemented in FLY for this occasion); the Stark broadening took care of the line merging naturally. The positions of the He-like satellites to the $\mathrm{H}$-like $2 p-1 s$ line were defined originally in FLY for carbon through interpolation from similar lines in other elements, and they appeared at wavelengths about $0.35 \AA$ lower than in the experimental spectra or the Kelly tables. We corrected this in the code to permit a better comparison with the experimental data. Figure 2 gives an example of a match for the lineout at $t=225 \mathrm{ps}$, and a comparison of artificial spectra obtained 


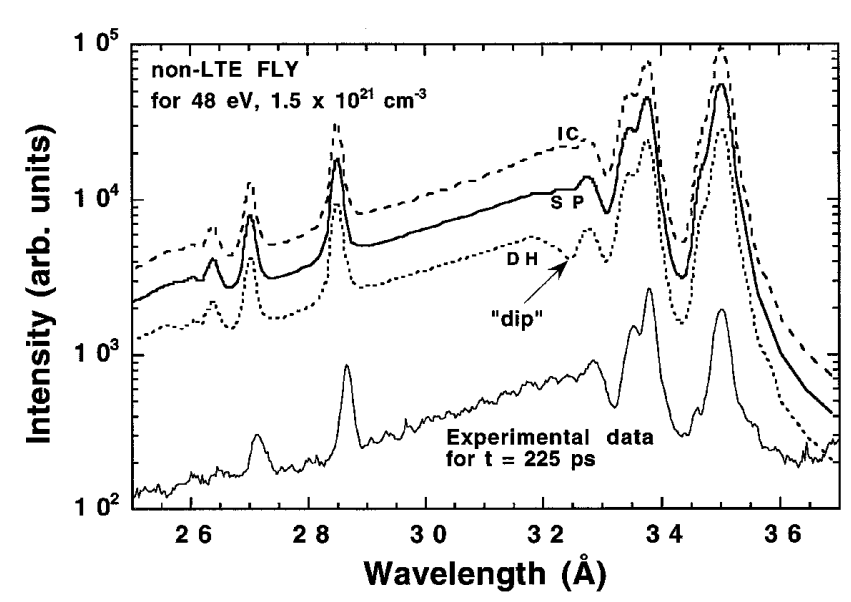

FIG. 2. Comparison of a lineout at $t=225 \mathrm{ps}$ and non-LTE FLY simulations at $48 \mathrm{eV}$ (determined from the slope of the continuum) and $1.5 \times 10^{21} \mathrm{~cm}^{-3}$. Three different artificial spectra are shown, corresponding to the cases where the continuum lowering is calculated with the ion-cell (IC, longdashed line), Stewart-Pyatt (SP, solid line), and Debye-Huckel (DH, short-dashed line) models. Note the conspicuous "dip" in the DH spectra near the He-like series limit. The lineouts are offset vertically for better viewing.

with the different pressure ionization models. While the ion-cell (long-dashed line) and Stewart-Pyatt (solid line) models give almost undistinguishable fits for similar densities, we found it impossible to properly match artificial spectra generated with Debye-Huckel pressure ionization (short dashes), regardless of the choice of density. The "dip" in the spectrum near the series limit for the DebyeHuckel case is caused by an overestimation of the continuum lowering, leaving no merged bound-bound transitions to contribute to the spectrum in that region. This disagreement - the first time one can spectroscopically differentiate between continuum lowering models - is due to the strongly coupled nature of the plasma which, as will come clear in the next figure, precludes the use of models based on the Debye-Huckel formalism.

The dynamics of the electron temperature and density is plotted in Fig. 3(a) for the first 225 ps of the emission; the Stewart-Pyatt pressure ionization model was used for the fits with FLY. A highest density of $5 \times 10^{22} \mathrm{~cm}^{-3}$ is inferred, which corresponds to one-tenth of the solid density for carbon with an average ionization $Z=4.5$. While the target and its emission did start at solid density, the 4-ps temporal resolution gives an averaged value of the plasma parameters over that time. Similarly, since the detected emission is integrated along the line of sight of the spectrometer, the measured plasma parameters are representative only of those plasma shells that are optically thin to the emissions. The temperature ranges from 50 to $80 \mathrm{eV}$, and this relatively low temperature with the high densities combine to produce the strong Coulomb coupling, as is evident in Fig. 3(b), which plots the coupling parameter $\Gamma$, and the number of particles in the Debye sphere, $N_{D}$. Up to $t \approx 30 \mathrm{ps}$, the Debye sphere contains less than one particle on average, and this number stays below
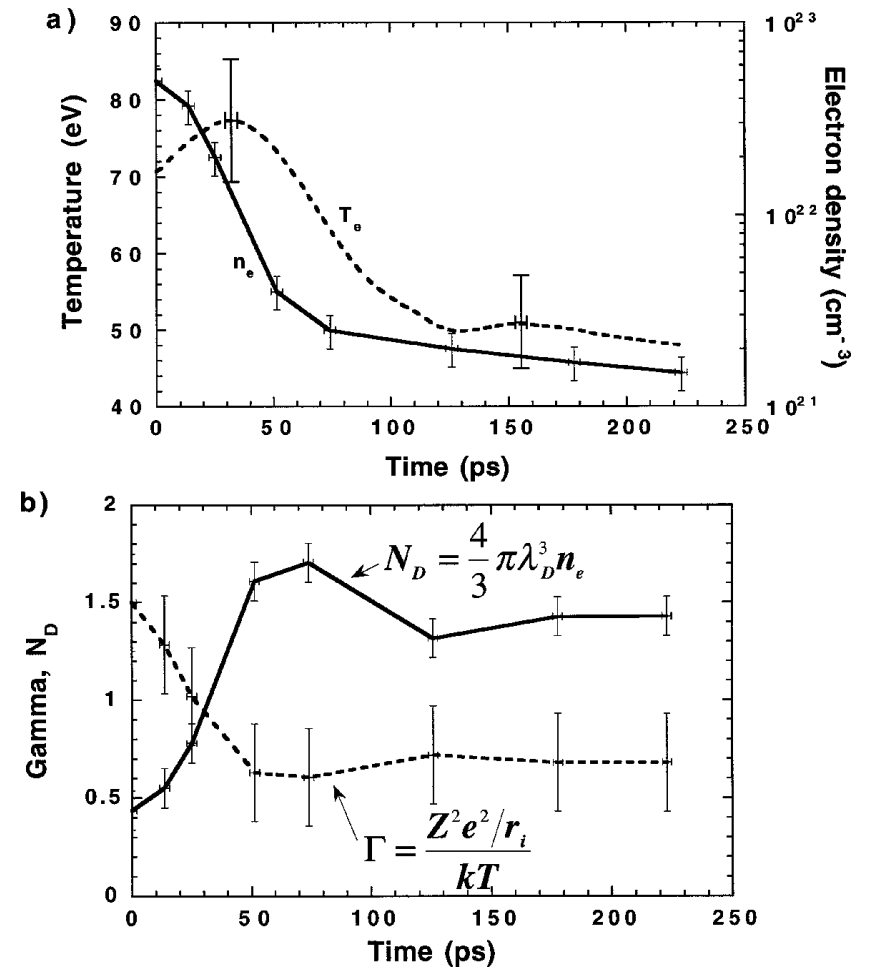

FIG. 3. Conditions as a function of time: (a) Electron density and temperature; (b) coupling parameter $\Gamma$ and number of particles in the Debye sphere $N_{D}$.

two in the first 225 ps. Similarly, the ratio of potentialto-kinetic energies hovers around one for the duration of the observation. Under these conditions, the concept of a Debye sphere has little statistical significance: The positive charges are not adequately shielded by electrons and Coulomb collisions dominate the ionized gas, which then cannot be rigorously referred to as a plasma. This was reflected in the pressure ionization, which cannot be described using the Debye-Huckel model (see Fig. 2). Other authors [21] could not spectroscopically differentiate between the three simple models of pressure ionization, possibly because of their lower plasma coupling $(\Gamma<0.2)$ for which the Debye-Huckel formalism is still at least marginally valid.

Figure 4 shows the dynamics of the experimentally detected series limit. This is compared to the series limit predicted by the Inglis-Teller (IT) model using the diagnostics from Fig. 3(a). On the left axis is the energy below the ionization potential (IP), and the corresponding principal quantum number $n$ are indicated on the right vertical axis. The uncertainty on the IT values corresponds to the energy difference between the last unmerged and the first merged levels. The error bars on the measured series limit includes the uncertainty in the position of the edge. A good agreement is found between the InglisTeller prediction and our detected series limit in these SCPs. This validation of the Inglis-Teller model using independent diagnostics suggests that it can be safely used as a density diagnostic in that plasma parameter range, 


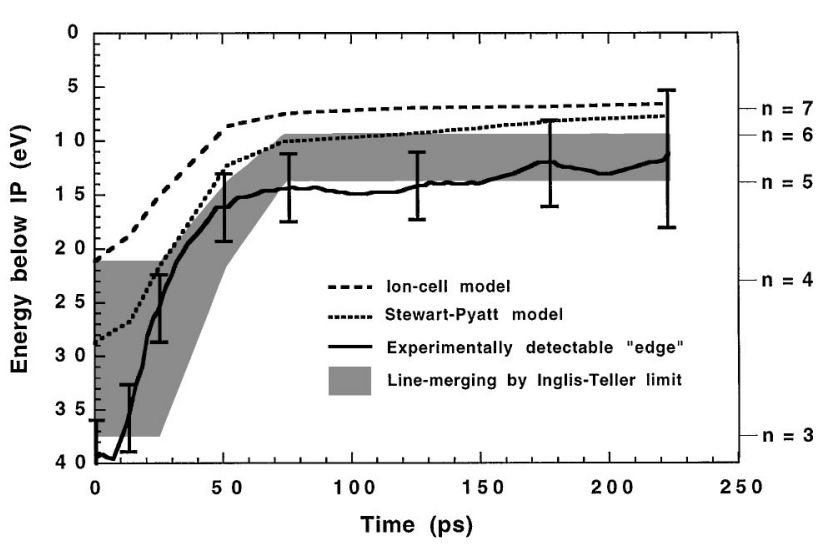

FIG. 4. Dynamics of the continuum lowering and line merging. The experimentally detectable series limit is plotted (solid line) with a 4 ps resolution and error bars at eight times $(0,14$, $25,50,75,125,175$, and 225 ps after the start of the emission). It is compared to that calculated with the Inglis-Teller limit using the densities and temperatures from Fig. 3(a). Also included are the prediction for the pressure ionization given by the ion cell and Stewart-Pyatt models. On the right vertical axis we give the position of the principal quantum number $n$ in He-like carbon.

but that temporal resolution is necessary for a meaningful result. We also show in Fig. 4 the pressure ionization predicted with the ion-cell (long dashed line) and StewartPyatt (short dashed line) models for the corresponding plasma conditions. To the best of our knowledge, this is the highest percentage of pressure ionization recorded to date in a laboratory plasma (with $7.3 \%$ of the IP at $t=0$ with the Stewart-Pyatt model). While the two models show some differences, particularly for the earlier times, they agree on the number of existing principal quantum numbers $n$ to within one throughout our parameter range. As expected, the continuum lowering is hidden by the line merging, and can therefore not be used as a density diagnostics on its own. Excited states with $n$ above the pressure ionization curves in Fig. 4 are considered free and part of the continuum, while those with $n$ below the Inglis-Teller predictions are bound. Levels between the pressure ionization and Inglis-Teller curves are broadened past merging, and are considered hybridized, i.e., they are bound but their wave functions are shared by more than one ion in the plasma [12].

In summary, we presented time- and space-resolved XUV spectra of carbon plasmas that were created by focusing 100-fs laser pulses on a solid target to an intensity of $10^{17} \mathrm{~W} / \mathrm{cm}^{2}$. The spectra were collected with a jitter-free $\mathrm{x}$-ray streak camera which conserved a temporal resolution of $4 \mathrm{ps}$ over the accumulation of 600 laser shots at a $10-\mathrm{Hz}$ repetition rate. We followed the transition of the target material as it went from being a strongly coupled Coulomb system, with less than one particle in the Debye sphere for the first 30 ps $(\Gamma \approx 1.5)$, to being a intermediately coupled plasma $(\Gamma \approx 0.5)$. When the data were compared to analytical and non-LTE FLY calculations, it was found that the Debye-Huckel model visibly breaks down in conditions of strong coupling, while the ion-cell and Stewart-Pyatt models gave good agreement with each other and the experimental spectra. The Inglis-Teller limit was validated over our plasma parameter range.

This work was done under the auspices of the Department of Energy, Contract No. DE-FG02-96ER14685, with facilities supported by the Center for Ultrafast Optical Science under NSF Grant No. STC PHY 8920108. M. Nantel is supported in part by the FCAR fund. We would also like to thank R.W. Lee, J. B. Workman, S. Pikuz, A. Maksimchuk, J.C. Kieffer, and G. Mourou for fruitful discussions, as well as D. Kaplan, T. Oksenhendler, J. Faure, and M. Finlayson for assistance with the experiment, and Spectrogon Corp., for the laser compressor gratings.

[1] S. Ichimaru, Rev. Mod. Phys. 54, 1017 (1982).

[2] C. M. Lee and A. Hauer, Appl. Phys. Lett. 33, 692 (1978); B. A. Hammel et al., Phys. Rev. Lett. 70, 1263 (1993).

[3] L. DaSilva et al., Phys. Rev. Lett. 62, 1623 (1989); A. Djaoui et al., Plasma Phys. Controlled Fusion 31, 111 (1989).

[4] E. Leboucher-Dalimier et al., J. Quant. Spectrosc. Radiat. Transfer 51, 187 (1994).

[5] J. Workman et al., Appl. Phys. Lett. 70, 312 (1997).

[6] H. R. Griem, Plasma Spectroscopy (McGraw-Hill, New York, 1964).

[7] P. G. Burkhalter et al., Rev. Sci. Instrum. 63, 5052 (1992); G. A. Kyrala et al., Appl. Phys. Lett. 60, 2195 (1992); D. Riley et al., Phys. Rev. Lett. 69, 3739 (1992).

[8] J. Itatani et al., Opt. Commun. (to be published).

[9] A. Maksimchuk et al., Rev. Sci. Instrum. 67, 697 (1996).

[10] C. Y. Côté et al., SPIE Proceedings Vol. 2869 (SPIEInternational Society for Optical Engineering, Bellingham, WA, 1997), p. 956.

[11] R. L. Kelly, J. Phys. Chem. Ref. Data Suppl. 1 16, 1 (1987). All atomic transition wavelengths and energies in the present paper are derived from Kelly's.

[12] D. G. Hummer and D. Mihalas, Astrophys. J. 331, 794 (1988); R. M. More, J. Quant. Spectrosc. Radiat. Transfer 27, 345 (1982); G. Massacrier, J. Quant. Spectrosc. Radiat. Transfer 51, 221 (1994).

[13] B. J. B. Crowley, Phys. Rev. A 41, 2179 (1990).

[14] J. Stein and D. Salzmann, Phys. Rev. A 45, 3943 (1992); M. W. C. Dharma-wardana and F. Perrot, Phys. Rev. A 45, 5883 (1992).

[15] L. G. D’yachkov et al., J. Phys. B 21, 1939 (1988).

[16] A. Unsold, Z. Astrophys. 24, 355 (1948).

[17] A. Klisnick et al., Appl. Phys. B 50, 153 (1990).

[18] R. W. Lee and J.T. Larsen, J. Quant. Spectrosc. Radiat. Transfer 56, 535 (1996).

[19] D. J. Heading et al., J. Quant. Spectrosc. Radiat. Transfer 54, 167 (1995); J. C. Stewart and K. D. Pyatt, Jr., Astrophys. J. 144, 1203 (1966); R. M. More, in Applied Atomic Collision Physics (Academic Press, New York, 1982), Vol. 2.

[20] D. R. Inglis and E. Teller, Astrophys. J. 90, 439 (1939).

[21] D. J. Heading et al., Phys. Rev. Lett. 74, 3616 (1995). 